\title{
The Distribution Pattern of Traditional Chinese Medicine Syndromes in 549 Patients with Type 2 Diabetes
}

\author{
Ge-Di Zhang ${ }^{1, *}$ \\ $\mathrm{Xi}-\mathrm{Xi} \mathrm{Liu}^{2, *}$ \\ Jia-Lang Liang ${ }^{3}$ \\ Qi-Ming $\mathrm{Hu}^{4}$
}

'Department of Endocrinology, Yuncheng Hospital of Traditional Chinese Medicine, Yuncheng, 274700, People's Republic of China; ${ }^{2}$ Department of Endocrinology, Yichun Hospital of Traditional Chinese Medicine, Yichun, 336000, People's Republic of China; ${ }^{3}$ Department of Endocrinology, Guangdong Integrated Traditional Chinese and Western Medicine Hospital, Foshan, 528000, People's Republic of China; ${ }^{4}$ Department of Endocrinology, Affiliated Hospital of Jiangxi University of Traditional Chinese Medicine, Nanchang, 330000, People's Republic of China

*These authors contributed equally to this work
Correspondence: Qi-Ming Hu Department of Endocrinology, Affiliated Hospital of Jiangxi University of Traditional Chinese Medicine, No. 445 of Bayidadao Street, Donghu District, Nanchang, 330000, People's Republic of China

Tel +8679107702803

Email huqimingqw_as@163.com
Objective: This study aimed to summarize the distribution pattern of traditional Chinese medicine (TCM) syndromes in patients with type 2 diabetes mellitus (T2DM).

Methods: The frequency, characteristics and distribution of all TCM syndromes of 549 patients with T2DM were analyzed.

Results: The average age of T2DM onset was higher in women than in men (ie, men experienced earlier onset). The distribution of TCM syndromes, in order of frequency, was as follows: damp-heat trapping spleen (including spleen deficiency and dampness, damp heat due to spleen deficiency, and qi weakness due to spleen deficiency) (58.29\%), qi-yin deficiency $(16.03 \%)$, deficiency of yin and excessive heat $(12.93 \%)$, blood stasis in collaterals $(9.41 \%)$, and yin-yang deficiency $(3.21 \%)$. The physical intensity of patients' occupational activity was mainly light $(49.6 \%)$, followed by heavy $(31.4 \%)$ and moderate $(19.0 \%)$.

Conclusion: Damp-heat trapping spleen is the most common TCM syndrome in patients with T2DM, with damp heat due to spleen deficiency the most significant subtype. This syndrome tends to occur in people over the age of 60 and those undertaking too much or too little physical activity in their occupational activities. The traditional "three more and one less" symptoms do not adequately describe the clinical symptoms of T2DM.

Keywords: type 2 diabetes, TCM syndrome differentiation, damp heat due to spleen deficiency, epidemiology, clinical symptoms

\section{Introduction}

With recent lifestyle changes, including improvements in material living standards and a decrease in physical labor tolerance, the incidence of type 2 diabetes mellitus (T2DM) - usually a geriatric disease - in the younger population is increasing. ${ }^{1,2}$ At the same time, the pressures of an aging population are growing and have become a major problem, ${ }^{3}$ endangering public health. T2DM belongs to the category of diabetes mellitus. ${ }^{4}$ Although developments in modern medical technology have led to a deeper understanding of T2DM, it is difficult to explain the pathogenesis ${ }^{5}$ of all cases of T2DM through the traditional Chinese medicine (TCM) concepts of yin deficiency and excessive heat. Based on clinical findings, many doctors have begun to attach importance to the role of the spleen in the pathogenesis of T2DM, proposing new views ${ }^{6}$ related to the symptom-complex of excessive eating. Dr. He Shaoqi He offered a theoretical point of view of the homology of the pancreas and spleen, ${ }^{7}$ suggesting that, although the pancreas has differences in 
internal and external secretion-ie, it internally releases insulin into the blood to promote the use of glucose and externally secretes pancreatic amylase for the digestion of starch, pancreatic lipase for the digestion of fat, and tryptase for the digestion of proteins, which enter the small intestine through the bile duct for the digestion of foodits processes should be classified in the spleen system ${ }^{7}$ of TCM, as both its exocrine and endocrine characteristics are related to the transportation, transformation, and decomposition of the essence of water and food under the function of the spleen and stomach. Prescriptions by Zhu Chenyu and Guan Youbo for eliminating the diabetes symptom of thirst have also embodied this theory and verified it. ${ }^{8} \mathrm{He}$ Xuewei and $\mathrm{Hu}$ Qiming used Qiwei Baizhu Powder to treat patients T2DM displaying spleen deficiency with dampness. The results after four weeks of treatment indicated that Qiwei Baizhu Powder can alleviate symptoms (depression, fatigue, and head and body distress), reduce fasting and postprandial blood glucose spikes, and increase the levels ${ }^{9}$ of glucagon-like peptide1 (GLP-1) in patients. This reflects the role of gastrointestinal hormones in regulating blood sugar, providing a new theoretical basis for spleen deficiency resulting in diabetes and greatly aiding the understanding ${ }^{10}$ of TCM in relation to $\mathrm{T} 2 \mathrm{DM}$.

With respect to the treatment of T2DM, TCM prescriptions for invigorating the spleen, resolving phlegm, tonifying the kidneys, and dehumidifying and activating the blood can help improve lipid metabolism, reduce fat accumulation in vivo, improve circulation, promote the use of nutrients in the body, increase the utilization of insulin in muscles and other cells, and reduce insulin resistance. ${ }^{3}$ This last improvement not only reduces blood sugar but also prevents the occurrence ${ }^{4}$ of complications, and it has a unique role to play in the treatment of T2DM in the field of TCM. The present study aims to summarize the distribution pattern of TCM syndromes in patients with T2DM in order to identify syndrome distribution patterns for T2DM. This will provide an epidemiological basis for the diagnosis and treatment of T2DM.

\section{Methods and Data}

\section{Patients Sample}

A total of 549 outpatients and inpatients (280 men and 269 women) who were admitted to the Department of Diabetes of the Affiliated Hospital of Jiangxi University of
Traditional Chinese Medicine between May 2015 and October 2016 met the diagnostic criteria for T2DM proposed by the World Health Organization's Committee of Experts on Diabetes (1999). The study was conducted in accordance with the Declaration of Helsinki (2013 revision) and approved by the Ethics Committee of the Affiliated Hospital of Jiangxi University of Traditional Chinese Medicine. Informed consent was obtained from all patients.

\section{Inclusion Criteria}

Patients were included who (1) met the diagnostic criteria of T2DM; (2) were between 20 and 79 years old; (3) had already received oral hypoglycemic drugs or insulin, or a combination of the two; (4) had no apparent organ dysfunction; and (5) volunteered to cooperate with the study and signed the informed consent form.

\section{Exclusion Criteria}

Patients were excluded who (1) had particular types of diabetes, including gestational diabetes or diabetes with pregnancy; (2) had experienced acute complications of diabetes (such as hyperosmolar coma, diabetic ketoacidosis, and diabetic lactic acidosis); (3) had used antipsychotic drugs, hormone drugs, or anti-obesity drugs within the last two weeks; (4) had malignant tumors, immune or blood system diseases, or other diseases; (5) had infectious diseases, stress diseases, or other acute diseases; (6) were addicted to alcohol or drugs; or (7) had objections to the collection of personal information or other relevant data.

\section{Additional Exclusion Criteria}

Also excluded were patients who (1) met the exclusion criteria after being included; (2) failed to cooperate with data collection related to the four diagnostic methods; (3) experienced cardiovascular and cerebrovascular diseases; or (4) had ketonic acidosis or other acute complications.

\section{Dropout Criteria}

Dropout occurred where (1) collection of relevant data in the research process did not meet the requirements for completeness; (2) results could not be analyzed due to incomplete information; or (3) patients were unwilling to continue the study. 


\section{Data Collection}

TCM Syndrome Diagnostic Criteria

For the patients who met the requirements, two trained professionals collected basic information and TCM symptoms for TCM syndrome differentiation and syndrome analysis using the Basic Information Collection Table of Type 2 Diabetes and Information Collection Table of Four Diagnostic Methods of TCM Symptoms. For TCM syndrome differentiation and classification, the patients who met the inclusion criteria were divided into five groups (based on the Clinical Guidelines of New Chinese Medicine). Syndrome type classification and diagnosis were based on the investigation results of an expert group, who used the State Administration of Traditional Chinese Medicine Clinical Research Guiding Principles for the Treatment of Diabetes with Traditional Chinese Medicine New Drugs (2002 edition), as follows:

1. Primary and secondary symptoms of yin deficiency and heat accumulation syndrome: three more and one less clinical features are relatively prominent; dry mouth, lips, and tongue, sore throat, uncomfortable gums, and skin infection. Tongue red, moss, less pulse string number and so on.

2. Primary and secondary symptoms of qi and yin deficiency syndrome: often accompanied by three more and one less symptoms or fat, physical exhaustion, and inability to bear weight. Tongue light red, tooth marks, pulse and so on.

3. Primary and secondary symptoms of blood stasis syndrome: skin is not wrong, hand and foot numbness (with or without limb pain), coronary artery injury, cerebral artery obstruction, or decline of vision/hearing. Tongue dark purple with stasis spots on the edges or a hypoglossal varicose vein, pulse string is fine or has fine acerbity.

4. Primary and secondary symptoms of yin and yang deficiency syndrome: dull and unclean complexion, atrophy of the wheel, frequent and cloudy urination or excessive urine volume, weakness in the waist and knees, impotence, thin stools, swelling, dizziness, and discomfort. Tongue dark red, light and fat, with less moss, heavy, weak pulse, etc.

5. Primary and secondary symptoms of damp-heat syndrome of spleen entrapment: heavy head pain, heavy body sleepiness, epigastric puffiness, thirst and drinking less, plump and tender tongue, yellow and greasy moss, smooth pulse string or moisten number

Description of symptom grade: see Annex 1.

\section{Classical of Occupational Physical Exertion}

Classification of the patients' occupational physical exertion followed Article 36 of the Labor Law of the People's Republic of China. Because there were very few patients in the very heavy labor category (IV), only levels I-III were used.

Level I: Eight-hour working days, average energy consumption value 3.5588 million joules/person, work time at a rate of $61 \%$; net work time 293 minutes, equivalent to light labor.

Level II: Eight-hour working days, average energy consumption value 5.5601 million joules/person, work time at a rate of $67 \%$; net work time 320 minutes, equivalent to moderate labor.

Level III: Eight-hour working days, average energy consumption value 7.3102 million joules/person, work time at a rate of $73 \%$; net work time 350 minutes, equivalent to heavy labor.

Level IV: Eight-hour working days, average energy consumption value 11.3044 million joules/person, work time at a rate of $77 \%$; net work time 370 minutes, equivalent to very heavy labor.

\section{Statistical Analysis}

SPSS 11.5 software was used to analyze the age, sex, course of disease, and physical labor intensity data in relation to all syndromes in order to investigate the syndrome distribution pattern for T2DM.

\section{Results}

\section{Age Distribution}

Table 1 shows that the 549 patients with T2DM were mainly aged $60-69(54.28 \%)$ or over 70 (41.17\%), these two groups accounting for $95.45 \%$ of the sample. However, $4.4 \%$ of the patients were under 40 years old.

\section{Distribution of TCM Syndromes}

Table 2 shows that the most common TCM syndrome among the 549 patients was damp heat due to spleen deficiency $(26.23 \%)$. This was followed by spleen deficiency with dampness (20.95\%), qi-yin deficiency 
Table I Gender Proportion of 549 T2DM Patients in Each Age Group

\begin{tabular}{|l|c|c|c|c|c|}
\hline \multirow{2}{*}{$\begin{array}{l}\text { Age } \\
\text { Groups }\end{array}$} & \multirow{N}{*}{} & \multicolumn{2}{|c|}{ Male } & \multicolumn{2}{c|}{ Female } \\
\cline { 2 - 6 } & & Cases & $\begin{array}{c}\text { Percentage } \\
\text { (\%) }\end{array}$ & Cases & $\begin{array}{c}\text { Percentage } \\
\text { (\%) }\end{array}$ \\
\hline $20-29$ & 8 & 4 & 1.43 & 4 & 1.49 \\
$30-39$ & 10 & 4 & 2.86 & 2 & 0.74 \\
$40-49$ & 91 & 60 & 21.43 & 31 & 11.52 \\
$50-59$ & 101 & 54 & 19.29 & 47 & 17.47 \\
$60-69$ & 188 & 78 & 27.86 & 110 & 40.89 \\
$\geq 70$ & 151 & 76 & 27.14 & 75 & 27.88 \\
Total & 549 & 280 & 100 & 269 & 100 \\
\hline
\end{tabular}

(16.03\%), deficiency of yin and excessive heat $(12.93 \%)$, qi weakness due to spleen deficiency $(11.11 \%)$, blood stasis in collaterals $(9.47 \%)$, and yinyang deficiency syndrome (3.28\%). As two of these syndromes - spleen deficiency with dampness and qi weakness due to spleen deficiency-are subtypes of damp-heat trapping spleen, this syndrome accounts for $58.29 \%$ of total cases (320 patients).

Table 2 also shows that, of the 71 patients with deficiency of yin and excessive heat, more were female (42) than male (29). The number of patients with yin deficiency and excessive heat was higher than those with qi-yin deficiency, as was the number of patients with qi weakness due to spleen deficiency.
Table 3 Classification of Occupational Labor Intensity of T2DM Patients

\begin{tabular}{|l|c|c|}
\hline Occupation & Cases & Percentage (\%) \\
\hline Mild manual workers & 293 & 53.37 \\
Moderate manual workers & 103 & 18.76 \\
Severe manual workers & 153 & 27.87 \\
Total & 549 & 100.00 \\
\hline
\end{tabular}

\section{Classification of Physical Intensity of Occupational Activity}

Table 3 shows that $53.37 \%$ of the patients undertook light labor, $18.76 \%$ undertook moderate labor, and $27.87 \%$ undertook heavy labor.

\section{Frequency of TCM Symptoms}

Table 4 shows how frequently different TCM symptoms occurred. The ten most common symptoms were thirst and drinking more $(66.12 \%)$, blurred vision $(62.48 \%)$, mental fatigue $(62.12 \%)$, dry mouth and throat $(61.75 \%)$, insomnia and drowsiness $(59.93 \%)$, fear of heat $(57.92 \%)$, tiredness and weakness (52.64\%), sweating (52.64\%), frequent night urination $(50.46 \%)$, and cold pain in the waist and knees (49.91\%). Neither the traditional symptoms of increased appetite, urination, thirst, and unexplained weight loss (three more and one less) nor yin deficiency and excessive heat can easily be generalized to the clinical symptoms of T2DM.

Table 2 General Condition of TCM Syndromes

\begin{tabular}{|l|c|c|c|c|c|c|}
\hline \multirow{2}{*}{ Syndromes } & \multicolumn{1}{|c|}{$\mathbf{N}$} & \multicolumn{2}{|c|}{ Male } & \multicolumn{2}{c|}{ Female } & \multicolumn{2}{c|}{$\begin{array}{c}\text { Median Age (Interquartile } \\
\text { Distance) }\end{array}$} \\
\cline { 3 - 6 } & & Cases & $\begin{array}{c}\text { Percentage } \\
\text { (\%) }\end{array}$ & Cases & $\begin{array}{c}\text { Percentage } \\
\text { (\%) }\end{array}$ & \\
\hline $\begin{array}{l}\text { Deficiency of yin and excessive heat } \\
\text { syndrome }\end{array}$ & $71(12.93 \%)$ & 29.00 & 10.36 & 42.00 & 15.61 & $64.00(14.00)$ \\
\hline Qi-yin deficiency syndrome & $88(16.03 \%)$ & 46.00 & 16.43 & 42.00 & 15.61 & $61.00(21.80)$ \\
\hline Syndrome of blood stasis in collaterals & $52(9.47 \%)$ & 24.00 & 8.57 & 28.00 & 10.41 & $63.00(18.30)$ \\
\hline Yin-yang deficiency syndrome & $18(3.28 \%)$ & 9.00 & 3.21 & 9.00 & 3.35 & $66.50(13.00)$ \\
\hline $\begin{array}{l}\text { Syndrome of spleen deficiency with } \\
\text { dampness }\end{array}$ & $144(26.23 \%)$ & 82.00 & 29.29 & 62.00 & 23.05 & $61.50(18.00)$ \\
\hline $\begin{array}{l}\text { Syndrome of dampness-heat due to } \\
\text { spleen deficiency }\end{array}$ & $115(20.95 \%)$ & 58.00 & 20.71 & 57.00 & 21.19 & $62.00(15.00)$ \\
\hline $\begin{array}{l}\text { Syndrome of qi weakness due to spleen } \\
\text { deficiency }\end{array}$ & $61(11.11 \%)$ & 32.00 & 11.43 & 29.00 & 10.78 & $64.00(21.50)$ \\
\hline
\end{tabular}


Table 4 TCM Symptom Count Table of 549 T2DM Patients

\begin{tabular}{|c|c|c|c|c|}
\hline Symptoms & Light (Cases) & Medium (Cases) & Heavy (Cases) & Percentage (\%) \\
\hline Mental fatigue & 150 & 102 & 89 & 62.12 \\
\hline Heaviness in head and drowsiness & 122 & 77 & 68 & 48.63 \\
\hline Upset & 83 & 81 & 77 & 43.9 \\
\hline Chest pain and chest distress & 74 & 89 & 18 & 32.97 \\
\hline Dry mouth and throat & 104 & 105 & 130 & 61.75 \\
\hline Abdominal distention & 45 & 59 & 51 & 28.23 \\
\hline Poor appetite & 82 & 52 & 29 & 29.69 \\
\hline Tiredness and weakness & 100 & 89 & 100 & 52.64 \\
\hline Intolerance of cold and clod limbs & 56 & 70 & 99 & 40.98 \\
\hline Fear of heat & 72 & 105 & $14 \mid$ & 57.92 \\
\hline Frequent urination at night & 140 & 101 & 36 & 50.46 \\
\hline Cold pain of the waist and knee & 67 & 100 & 107 & 49.91 \\
\hline Numbness of limb & 78 & 110 & 74 & 47.72 \\
\hline Limb pain & 77 & 68 & 30 & 31.88 \\
\hline Limb spasm & 70 & 107 & 55 & 42.26 \\
\hline Dizziness & 83 & 80 & 43 & 37.52 \\
\hline Thirst and drinking more & 125 & 105 & 133 & 66.12 \\
\hline Dark purple lips & 124 & 85 & 24 & 42.44 \\
\hline Increased eating with rapid hungering & 60 & 64 & 52 & 32.06 \\
\hline Shortness and laziness to speak & 87 & 49 & 42 & 32.42 \\
\hline Burning sensation of five centers & 23 & 32 & 13 & 12.39 \\
\hline Palpitation & 64 & 80 & 42 & 33.88 \\
\hline Insomnia and dreaminess & 76 & 96 & 157 & 59.93 \\
\hline Blurred vision & $|3|$ & 92 & 120 & 62.48 \\
\hline Spontaneous sweating & 89 & 49 & 151 & 52.64 \\
\hline Night sweat & 71 & 37 & 16 & 22.59 \\
\hline Cloudy and foamy urine & 74 & 85 & 67 & 41.17 \\
\hline Urgent urination and short duration & 106 & 78 & 26 & 38.25 \\
\hline Constipation & 42 & 73 & 86 & 36.61 \\
\hline Loose stool & 64 & 38 & 20 & 22.22 \\
\hline Unwell defecation & 60 & 56 & 84 & 36.43 \\
\hline Facial and limb swelling & 43 & 49 & 27 & 21.68 \\
\hline Squamous and dry skin & 73 & 34 & 29 & 24.78 \\
\hline
\end{tabular}


Table 4 (Continued).

\begin{tabular}{|l|c|c|c|c|}
\hline Symptoms & Light (Cases) & Medium (Cases) & Heavy (Cases) & Percentage (\%) \\
\hline Depression and frequent sigh & 49 & 59 & 69 & 32.24 \\
\hline Worry beyond measure & 81 & 85 & 91 & 46.81 \\
\hline Loss of weight & 107 & 73 & 70 & 45.54 \\
\hline
\end{tabular}

\section{Mean Age of T2DM Onset in Men and Women}

Table 5 shows that the mean onset age of T2DM was 59.68 years for male patients and 62.35 years for female patients. The average onset age was lower for male patients.

\section{Discussion}

T2DM has become a major problem ${ }^{11}$ in public health. Although developments in modern medical technology have facilitated a deeper understanding of T2DM, yin deficiency and excessive heat syndrome are unable to fully explain the pathogenesis of all T2DM cases. TCM prescriptions, specific to the treatment of T2DM, for invigorating the spleen, resolving phlegm, tonifying the kidneys, and dehumidifying and activating the blood can help improve lipid metabolism, reduce fat accumulation in vivo, improve circulation, promote the use of nutrients in the body, increase the utilization of insulin in the muscles and other cells, and reduce insulin resistance. ${ }^{12}$ The reduction of insulin resistance not only reduces blood sugar but also prevents the occurrence $^{13}$ of complications. It has a unique role to play in the treatment of T2DM in the field of TCM.

The present study found that the traditional "three more and one less" symptoms fail to adequately describe the characteristics of T2DM. In this study, the ten most common symptoms experienced by patients with T2DM were thirst and drinking more, blurred vision, mental fatigue, dry mouth and throat, insomnia and drowsiness, fear of heat, tiredness and weakness, sweating, frequent night urination, and cold pains in the waist and knees. He et $\mathrm{al}^{11}$ indicated that the most common TCM syndromes were spleen-kidney yang deficiency and damp-heat phlegm stasis (accounting for 30.47\% of the study population); qi-yin deficiency syndrome was found in another 69 patients (11.41\%). Wang et al, ${ }^{12}$ however, found that qi-yin deficiency occurred most frequently and that the frequency of damp-heat trapping spleen was not significant. In the TCM view of diabetes, yin deficiency and excessive heat do not occupy a dominant position in clinical practice ${ }^{14-18}$ instead, the spleen is seen as the main mover of the disease, the qi as the main rise, the stomach as the main receptor, and the decline to shun. Heat and humidity are stuck in the body and thus cause the epigastric discomfort and also nausea. In TCM, it's considered that spleen mainly controls muscles and limbs, so when heat and humidity are stuck in the spleen, the limbs would be affected as well. When heat and humidity accumulate in the spleen and stomach, fumigation can relieve the liver and gallbladder.

The present study found that people who undertake too much or too little physical intensity in their occupational activities are more likely to develop T2DM. Like previous studies, it was found that the prevalence of obesity, hypertension, and T2DM increased ${ }^{19,20}$ with a decline in physical activity. This is because physical activity can effectively improve insulin resistance and strengthen the

Table 5 Mean Age of Onset in Men and Women Among 549 T2DM Patients (Statistical Description)

\begin{tabular}{|c|c|c|c|c|c|}
\hline Age Groups & Number & Minimum (M) & Maximum (X) & Mean (E) & Standard Deviation \\
\hline \multicolumn{6}{|l|}{ Female } \\
\hline Age & 269 & 23.0 & 80.0 & 62.346 & 10.7459 \\
\hline Course of Disease (year) & 268 & 0 & 30 & 7.35 & 5.688 \\
\hline Effective N (column) & 268 & & & & \\
\hline \multicolumn{6}{|l|}{ Male } \\
\hline Age & 280 & 25.0 & 80.0 & 59.682 & 12.6540 \\
\hline Course of Disease (year) & 280 & 0 & 40 & 6.66 & 6.559 \\
\hline Effective $\mathrm{N}$ (column) & 280 & & & & \\
\hline
\end{tabular}


uptake of glucose by target cells, thereby reducing blood sugar and reversing type 2 prediabetes. Moderate physical labor is therefore of great significance for the prevention and treatment of T2DM. In this study, however, the number of patients undertaking heavy labor was 153-higher than the number of patients undertaking moderate labor (103) - which raises a question about the extent to which an increase in physical labor can improve the prevention of T2DM. Due to the limits of time and sample size in the present study, more data are needed to clarify this point.

\section{Conclusion}

In the TCM syndrome distribution in patients with T2DM, damp-heat trapping spleen is the most commonly experienced syndrome, with damp heat due to spleen deficiency the most common subtype. T2DM tends to occur in older people, with patients over 60 accounting for $95.45 \%$ of the cases included in this study. People undertaking too much or too little physical intensity in their occupational activities are more likely to experience T2DM. The traditional "three more and one less" symptoms do not adequately describe the clinical symptoms of T2DM.

\section{Acknowledgments}

We are particularly grateful to all the people who have given us help on our article.

\section{Funding}

This study was funded by the Jiangxi Provincial Health and Family Planning Commission Chinese Medicine Research Project (2014A055). The funding body had no role in the design of the study and collection, analysis, and interpretation of data and in writing the manuscript.

\section{Disclosure}

The authors declare that they have no competing interests.

\section{References}

1. Chinese Medical Association of Diabetes. China type 2 diabetes prevention and treatment guidelines 2013. Chin J Diab. 2014; 6:447-497.

2. Yang W, Lu J, Weng J, et al. Prevalence of diabetes among men and women in China. $N$ Engl J Med. 2010;362(12):1090-1101. doi:10. 1056/NEJMoa0908292

3. Xu Y, Wang L, He J, et al. Prevalence and control of diabetes in Chinese adults. JAMA. 2013;310(9):948-959. doi:10.1001/jama.2013.168118

4. Ji L, Tong X, Wang H, et al. Efficacy and safety of traditional Chinese medicine for diabetes: a double-blind, randomised, controlled trial. PLoS One. 2013;8(2):e56703. doi:10.1371/journal.pone.0056703
5. Xiao E, Luo L. Alternative therapies for diabetes: a comparison of Western and Traditional Chinese Medicine (TCM) approaches. Curr Diabetes Rev. 2018;14(6):487-496. doi:10.2174/157339981366617 0519103230

6. Feng Y, Fang Y, Wang Y, Hao Y. Acupoint therapy on diabetes mellitus and its common chronic complications: a review of its mechanisms. Biomed Res Int. 2018;2018:3128378. doi:10.1155/ 2018/3128378

7. Xie W, Du L. Diabetes is an inflammatory disease: evidence from traditional Chinese medicines. Diabetes Obes Metab. 2011;13(4): 289-301. doi:10.1111/j.1463-1326.2010.01336.x

8. Ai X, Yu P, Hou Y, et al. A review of traditional Chinese medicine on treatment of diabetic retinopathy and involved mechanisms. Biomed Pharmacother. 2020;132:110852. doi:10.1016/j.biopha.2020.110852

9. Li C, Zhang H, Li X. The mechanism of traditional Chinese medicine for the treatment of obesity. Diabetes Metab Syndr Obes. 2020; 13:3371-3381. doi:10.2147/DMSO.S274534

10. Yang R, Liu C, Li Q, et al. Artificial intelligence based identification of the functional role of hirudin in diabetic erectile dysfunction treatment [published online ahead of print, 2020 Oct 11]. Pharmacol Res. 2020:105244. doi:10.1016/j.phrs.2020.105244.

11. He WJ, Cao DM, Chen YB, et al. Explore of the beneficial effects of Huang-Lian-Jie-Du Decoction on diabetic encephalopathy in $\mathrm{db} / \mathrm{db}$ mice by UPLC-Q-Orbitrap HRMS/MS based untargeted metabolomics analysis [published online ahead of print, 2020 Sep 28]. J Pharm Biomed Anal. 2020;192:113652. doi:10.1016/j.jpba.2020.113652

12. Wang PP, Ke CM, Yao DY, et al. A cohort study on associations between fundus/intraocular pressure abnormality and medical check-up items [published online ahead of print, 2020 Oct 13]. Curr Eye Res. 2020:1-6. doi:10.1080/02713683.2020.1826978.

13. Wang L, Li S, Wang L, et al. Uncovering the protective mechanism of Taohong Siwu decoction against diabetic retinopathy via HIF-1 signaling pathway based on network analysis and experimental validation. BMC Complement Med Ther. 2020;20(1):298. doi:10.11 86/s12906-020-03086-0

14. An X, Jin D, Duan L, et al. Direct and indirect therapeutic effect of traditional Chinese medicine as an add-on for non-proliferative diabetic retinopathy: a systematic review and meta-analysis. Chin Med. 2020;15:99. doi:10.1186/s13020-020-00380-4

15. Zheng Y, Ding Q, Zhang L, et al. The effect of traditional Chinese medicine on gut microbiota in adults with type 2 diabetes: a protocol for systematic review and meta-analysis. Medicine. 2020;99(38): e22233. doi:10.1097/MD.0000000000022233

16. Huang X, Zhang M, Wu H, Wang X, Xu F. Study on the active ingredients and potential targets of rice bran petroleum ether extracts for treating diabetes based on network pharmacology [published online ahead of print, 2020 Aug 21]. Comb Chem High Throughput Screen. 2020. doi:10.2174/1386207323999200821162307

17. Cui FQ, Gao YB, Wang YF, et al. Effect of Tang-Shen-Ning decoction on podocyte epithelial-esenchymal transformation via inhibiting Wnt/ $\beta$ catenin pathway in diabetic mice [published online ahead of print, 2020 Aug 24]. Ann Palliat Med. 2020:apm-20-602. doi:10.21 037/apm-20-602.

18. Sha JY, Liu H, Xie YM, Zhuang Y. Clinical application characteristics of Ciwujia Injection in treatment of 5904 cases with cardiovascular disease in real world. Zhongguo Zhong Yao Za Zhi. 2020;45 (15):3525-3532. doi:10.19540/j.cnki.cjcmm.20200616.501.Chinese

19. Zheng WJ, Ren YS, Wu ML, et al. A review of the traditional uses, phytochemistry and biological activities of the Melastoma genus. J Ethnopharmacol. 2021;264:113322. doi:10.1016/j.jep.2020.113322

20. Song YJ, Wang X, Li XJ, Zhang ZL. Effect of Tiaoli Piwei needling technique on diabetic gastroparesis and transmembrane protein 16A. Zhongguo Zhen Jiu. 2020;40(8):811-815. doi:10.13703/j.0255-2930. 20190618-0001.Chinese 
Diabetes, Metabolic Syndrome and Obesity: Targets and Therapy is an international, peer-reviewed open-access journal committed to the rapid publication of the latest laboratory and clinical findings in the fields of diabetes, metabolic syndrome and obesity research. Original research, review, case reports, hypothesis formation, expert opinion and commentaries are all considered for publication. The manuscript management system is completely online and includes a very quick and fair peer-review system, which is all easy to use. Visit http://www.dovepress.com/testimonials.php to read real quotes from published authors. 